\title{
El papel del comercio en la revitalización de los centros urbanos en Europa: las declaraciones de Málaga y Lille
}

\section{Rafael Gómez Pascual *}

Europa es un continente esencialmente urbano. En las ciudades europeas se concentra más del $80 \%$ de la población residente en los 15 Estados de la Unión, siendo ellas los núcleos de desarrollo económico, social y cultural del Viejo Continente. Alrededor de 300 millones de ciudadanos de la Unión Europea habitan en áreas urbanas, y es básicamente en ellas donde han venido realizando sus actividades de aprovisionamiento de todo tipo de bienes - alimentos, ropa, libros, equipamiento de hogar, etc.- - Pero, como indica el Libro Verde del Comercio, elaborado por la Comisión a principios de 1997, «en la época del consumo de masas, las nuevas formas de distribución ban cambiado el uso del suelo urbano con la aparición de grandes centros comerciales fuera de las ciudades, asi como con la implantación muy extendida de tiendas especializadas a lo largo de los ejes de transporte, que desencadenan o contribuyen al declive económico de los centros urbanos. De becho, la planificación urbanística de los centros de las ciudades y el equilibrio comercial en las zonas urbanas constituyen cuestiones politicas importantes en muchos países».

La modificación del uso de los centros urbanos, cada vez más orientados hacia el sector de servicios, servicios fundamentalmente de oficina, financieros, de ocio, etc., y el encarecimiento paralelo del suelo, han dado lugar a un desplazamiento de la población hacia la periferia, un envejecimiento de la población residente en estas zonas y una progresiva reducción de la actividad comercial.

$Y$ ello da lugar a que muchos centros urbanos se queden vacíos cuando acaba la jornada laboral, cuando las oficinas cierran. Esos centros han perdido el atractivo comercial y se produce una reacción en cadena que hace que esa pérdida de atractivo acelere el deterioro de esas zonas que en épocas recientes eran el núcleo de la vida de la ciudad. A la pérdida de atractivo comercial se une una pérdida de atractivo global de la zona fuera de las horas centrales del día, con un incremento de la inseguridad ciudadana y una paulatina desatención municipal en aspectos tales como la iluminación, la limpieza, el mobiliario urbano, etc., que a su vez acelera el proceso.

Resta, no obstante, en esos centros urbanos una población residente, en general envejecida y con un incremento importante de los hogares unipersonales, que difícilmente encuentra en el comercio de proximidad satisfacer sus necesidades mínimas de aprovisionamiento, consecuencia de la desaparición de establecimientos comerciales motivada por una demanda efectiva baja. Debemos aquí volver a referirnos al Libro Verde del Comercio: el envejecimiento de la población europea es un hecho incuestionable, y para las personas ancianas y las que viven solas, las tiendas, sobre todo las de barrio, pueden ser la única posibilidad babitual de entrar en contacto y comunicar con otras personas y con el mundo exterior.

Cada vez es mayor el reconocimiento del importante papel desempeñado por el comercio en el mantenimiento de una vida comunitaria equilibrada —indica también el Libro Verde-, ya sea en el centro de las ciudades o en las tiendas de barrio, tanto en las zonas urbanas como en las rurales. Un centro comercial de barrio con suficiente vida suele funcionar también como centro cultural de la comunidad, confiriendo asi al comercio una importante función de cohesión social. Las tiendas, grandes o pequeñas, 
dan vida a los centros de las ciudades y frenan la creciente tendencia a la desertización urbana, que constituye un acicate para la delincuencia.

El origen de estos cambios en el uso y configuración del tejido urbano habría que buscarlo en los cambios evidentes en los hábitos y en la estructura de la población de las ciudades.

Los fenómenos migratorios, que propiciaban la concentración de la población en la periferia de las grandes ciudades, con un suelo más barato y mayor densidad de edificación, el desplazamiento de las nuevas familias que se van formando desde el centro hacia la periferia, la mejora de los medios de conservación de alimentos frescos, la incorporación de la mujer al trabajo y el aumento de la motorización, fenómenos estos más recientes en el sur del continente, han dado lugar a unos cambios en los hábitos de compra de los consumidores, que se traducen en una cada vez mayor ampliación de los plazos de compra y en una búsqueda de áreas que puedan satisfacer una gama muy amplia de necesidades, que además tratan de unir a otras actividades lúdicas, $y$, en definitiva, reduciendo la compra diaria de todo tipo de productos.

Todo ello ha dado lugar a la aparición y desarrollo de nuevas formas comerciales, que han supuesto un reto muy importante para el comercio tradicional, que en muchos casos no ha sido capaz de superar, modificando sustancialmente la estructura del sector del comercio minorista, con la aparición de nuevas empresas, más grandes y con una gestión moderna, capaces de atraer a un gran número de consumidores, en detrimento de la cuota del pequeño comercio tradicional.

Si a esto unimos el desarrollo de una red de transporte eficiente en todo el continente, y especialmente en las inmediaciones de sus grandes ciudades, que facilita el acceso a amplias zonas situadas fuera de los núcleos urbanos tradicionales, con grandes facilidades de aparcamiento y suelo barato, la aparición de nuevas fórmulas comerciales no se queda sólo en eso, sino que ha supuesto también el desplazamiento de estos nuevos establecimientos comerciales desde el centro a la periferia de las ciudades, fenómeno éste que empezó a ser importante en el Norte de Europa a partir de los años 1960, y que ha llegado hasta los países del Sur a partir de 1980, caracterizándose en ambos casos por una importante pérdida de cuota de mercado del «comercio de calle».

Nos encontramos ya con un enfrentamiento no de formatos o de dimensión empresarial, sino de localización de la actividad comercial que constituye el principal centro de atracción de la población consumidora.

El aumento del comercio en las zonas periféricas, en detrimento de los centros históricos y barrios de las ciudades, vuelve a ser motivo de reflexión de la Comisión Europea, que ya señalaba en el Libro Verde que una politica integrada a favor de la ciudad parece cada vez mas necesaria, y que no se trata de poner arbitrariamente unas formas de comercio en contra de las otras, sino de alcanzar un equilibrio que tenga en cuenta los intereses $y$ necesidades de todos los babitantes de las zonas urbanas $y$, de becho, de la sociedad en general, así como de garantizar que el acceso al mercado sigue siendo libre y que se mantiene una competencia activa.

Igualmente recomienda, ante el aumento del comercio en las zonas periféricas, no sólo estudiar la competencia entre pequeños comerciantes en los centros de las ciudades y los supermercados periféricos, sino también entre los grandes centros comerciales de las afueras y las tiendas grandes del centro, los supermercados especializados que no venden productos alimenticios, los pequeños comerciantes, etc., y observar la competencia entre polos comerciales más que la existente entre formas de comercio. Y a este respecto debemos añadir que toda fórmula comercial es válida y puede ocupar una cuota de mercado, si sabe adaptarse a los nuevos tiempos y estar en consonancia con lo que piden los consumidores.

Ante este estado de cosas, y con la opinión compartida por las administraciones de la Comunidad y de sus Estados miembros de la existencia de un problema cierto de desarmonización del entramado urbano, consecuencia del deterioro no deseado de ese poder de cohesión del tejido social y del territorio que lo estructura que corresponde al comercio, y que a su vez se configura como un elemento de atracción de otras actividades complementarias, cada vez más demandadas por la sociedad, como son el ocio, la cultura y la restauración, se propuso la celebración del Primer Congreso Europeo de Comercio y Ciudad.

Este Congreso se planteó con la finalidad de llevar a cabo una reflexión conjunta, en busca de soluciones a estos problemas, no únicamente con la finalidad de reunir a unos expertos para llevar a cabo este necesario debate abierto, sino, además, con el claro propósito de implicar y sensibilizar a todas las administraciones, especialmente a los ayuntamientos, sobre la importancia evidente de atender a estas cuestiones relativas a la mejora del comercio sobre el medio urbano - su medio natural-, no sólo a través de los propios mecanismos del planeamiento, sino también mediante el apoyo a realizaciones concretas.

Así las cosas, la iniciativa tomada por la antigua Dirección General de Comercio Interior, del Ministerio de Economía y Hacienda, encontró desde el primer momento el respaldo de la Comisión Europea, a través de la Dirección General XXIII y de la DG XVI, así como por el Comité de Regiones y por multitud de Organismos e Instituciones, entre los que cabe destacar al Consejo Superior de Cámaras de Comercio de España.

Se trataba de establecer un diálogo que contribuyera al mejor funcionamiento del mercado interior $\mathrm{y}$, especialmente, a la 
mejor comprensión de lo que debería ser el comercio del siglo XXI en su ámbito natural, que no es otro que el urbano. Se pretendió, y consiguió, provocar un amplio debate sobre la ciudad y la actividad comercial que ella genera, destacando con ello la importancia del sistema de ciudades en Europa, las regiones y la necesidad de su ordenación territorial. Se perseguía también, de acuerdo con el Libro Blanco del Comercio de las Comunidades Europeas, conseguir «garantizar que la situación del comercio se comprenda completamente en los debates sobre futuras iniciativas de politica comunitaria», pues «una clave para rentabilizar al máximo los actuales instrumentos comunitarios consiste en aumentar la sensibilidad de los distintos responsables sobre el estado del comercio», ya que ureforzar el conocimiento del sector no significa tomar medidas legislativas bruscas, sino más bien prestar una cuidadosa atención a la comunicación entre el comercio y las políticas comunitarias»

En el desarrollo de este primer congreso, que tuvo lugar en Torremolinos, del 24 al 26 de febrero de 1999, se debatieron diversos aspectos comunes al comercio y al entorno urbano, tales como los que se refieren a la conservación del patrimonio cultural, el medio ambiente, la rehabilitación de zonas degradadas, la mejora de las condiciones de accesibilidad, la cooperación entre empresas, la interacción entre el comercio y el turismo, la cooperación pública y privada y los aspectos legislativos y fiscales que les afectan.

Tras estos tres días de debates, se llegó a lo que se ha dado en llamar «Declaración de Málaga», que en 19 puntos sintetiza una propuesta de acción, que se puede estructurar en varios grandes grupos de preocupación que son:

- Mejora de la accesibilidad global a los centros urbanos, mediante la dotación de aparcamientos, fomento y mejora del transporte público y diversas medidas sobre el tráfico. El desarrollo de una vasta red de autopistas y carreteras de gran capacidad ha propiciado el desarrollo de las grandes concentraciones comerciales periféricas, que se han beneficiado de importantes inversiones públicas para convertirse en núcleos de atracción de consumidores. Se trata, en cierto modo, de compensar a los centros urbanos de este desequilibrio dotacional, en cuanto a accesos, para que también el consumidor que quiera llegar al comercio de centro ciudad, de cualquier dimensión o tipología, lo encuentre cómodo, fácil y asequible.

- Promoción de la diversidad de usos del centro urbano, ya que es tanto más completo y atractivo cuanto más diverso sea. Uno de los grandes atractivos de los espacios comerciales periféricos es el unir en un mismo lugar servicios de diferente índole, como son los comerciales, de restauración y de ocio. Favorecer y combinar esta diversidad en el centro urbano, de manera que el visitante pueda satisfacer en él todas sus necesidades, se muestra como una forma de atraer potenciales compradores o usuarios a los centros tradicionales de las ciudades, aumentando el atractivo global de los mismos. Además, la integración del tejido residencial en los centros urbanos, reteniendo al mayor número posible de población residente, favorecerá el mantenimiento de un tipo de comercios que la «terciarización» del centro ha desplazado.

- Necesidad de una coordinación en la toma de decisiones y en la planificación urbanística, de forma que ésta se realice bajo una visión integrada. Son diversos las administraciones y agentes implicados en el desarrollo urbano, por lo que éste no puede realizarse de espaldas a los agentes sociales que participan en la actividad del centro ciudad. Las Cámaras de Comercio y las Asociaciones de Comerciantes deben participar más activamente en los fenómenos de renovación urbana.

- Necesidad de una gestión integrada que permita coordinar los intereses de los distintos agentes, pero que también suponga un impulso activo para realizar acciones imprescindibles para los cambios cualitativos necesarios. Los centros urbanos deben estar gestionados con modelos similares a los de un centro comercial de la periferia, es decir, con un gerente, a ser posible único. Se trata de transformar los centros urbanos en centros comerciales abiertos, con una gestión unitaria, impulsando la figura del Gerente de Centro Urbano, utilizando el modelo de los town centre management, habituales en el Reino Unido.

Este gerente de centro urbano, o los centros de gestión colectiva de centro ciudad, utilizarán técnicas similares a los de los centros comerciales, incidiendo especialmente en la promoción y el marketing, prestando especial atención a la mejora de la seguridad, como elemento imprescindible para hacer atractivos los centros de ciudad, al igual que a la mejora de la limpieza e higiene y, en general de todo el entorno urbano, especialmente en su mobiliario, equipándole adecuadamente.

La promoción y publicidad, para atraer visitantes de otras áreas, ofreciendo una imagen unitaria y organizando actos públicos, culturales o festivos, es también una función a realizar por la gerencia unitaria del centro de ciudad.

- Potenciar la mejora de la calidad y el servicio como forma indispensable de recobrar la identidad de los centros urbanos, estableciendo con ello un mecanismo diferenciador de otras áreas de centralidad de nuevo cuño. Es aquí donde el comerciante, individualmente, debe actuar para ser competitivo, en un mercado donde los hábitos de consumo y los gustos de los consumidores evolucionan constantemente, a los que deberá adaptarse, ofreciéndole unos servicios diferenciados respecto a otros formatos y al comercio de otras áreas. El atraer visitantes al centro ciudad - potenciales compradores - no es suficiente si los distintos establecimientos no se muestran también atractivos, lo que servirá además para potenciar el atractivo global de ese centro. 
- Mejora de la financiación, buscando no sólo las mejores condiciones, sino vías alternativas de colaboración de los particulares en el proceso inversor y en el sostenimiento de los gastos. La cooperación entre las distintas administraciones, los agentes sociales y los propios comerciantes, el partenariado, se muestra indispensable para garantizar el éxito de operaciones de rehabilitación comercial.

- Mejora de la relación de las actividades turísticas con los cascos históricos y el tejido comercial. La rehabilitación de edificios singulares y la conservación y potenciación del patrimonio cultural de los centros históricos es, sin duda, un elemento favorecedor del aumento del tránsito de visitantes - potenciales compradores- de esa zona.

- Y, finalmente, el mantenimiento de la dimensión europea de estos fenómenos, pues, al fin y al cabo, la problemática de las ciudades es relativamente coincidente en todo este territorio $y$, por lo tanto, el traslado de las experiencias y de los distintos procesos entre las distintas regiones y ciudades puede resultar muy provechosa.

Esta última propuesta fue aceptada en su día por la Dirección General XXIII, y posteriormente asumida por la Dirección General de Empresas de la Comisión Europea, así como por la Dirección General de Empresas Comerciales, Artesanales y de Servicios de Francia, que ya en la clausura en Torremolinos asumió la responsabilidad de organizar el Segundo Congreso Europeo de Comercio y Ciudad, coincidiendo con el semestre de su presidencia del Consejo.

De esta forma, se organizó por Francia el Segundo Congreso Europeo de Comercio y Ciudad, que tuvo lugar en Lille durante los días 15 a 17 de noviembre de 2000 , como prolongación del celebrado en Málaga casi dos años antes. En palabras del Comisario europeo Erkki Likanen, en Málaga se establecieron los conceptos, las 19 proposiciones de su Declaración sobre las que apoyar la política a seguir en los dominios tan estrechamente ligados de Comercio y Ciudad. La iniciativa en Lille tiene por objeto dar a conocer «Las Buenas Prácticas» europeas en cuanto a estrategias eficaces de ronovación urbana puestas en práctica en el ámbito del «partenariado» entre las autoridades públicas y las colectividades locales.

Un instrumento metodológico del congreso ha sido el benchmarking - valoración de objetivos a alcanzar-: poner de manifiesto estrategias comerciales competitivas recientes desarrolladas en el medio urbano, puestas al día y valorizadas como instrumento útil y enriquecedor para los Estados miembros al poder aprender los unos de los otros en cuanto a los enfoques de los problemas comerciales y urbanos. El bencbmarking es un instrumento para identificar las mejores prácticas.

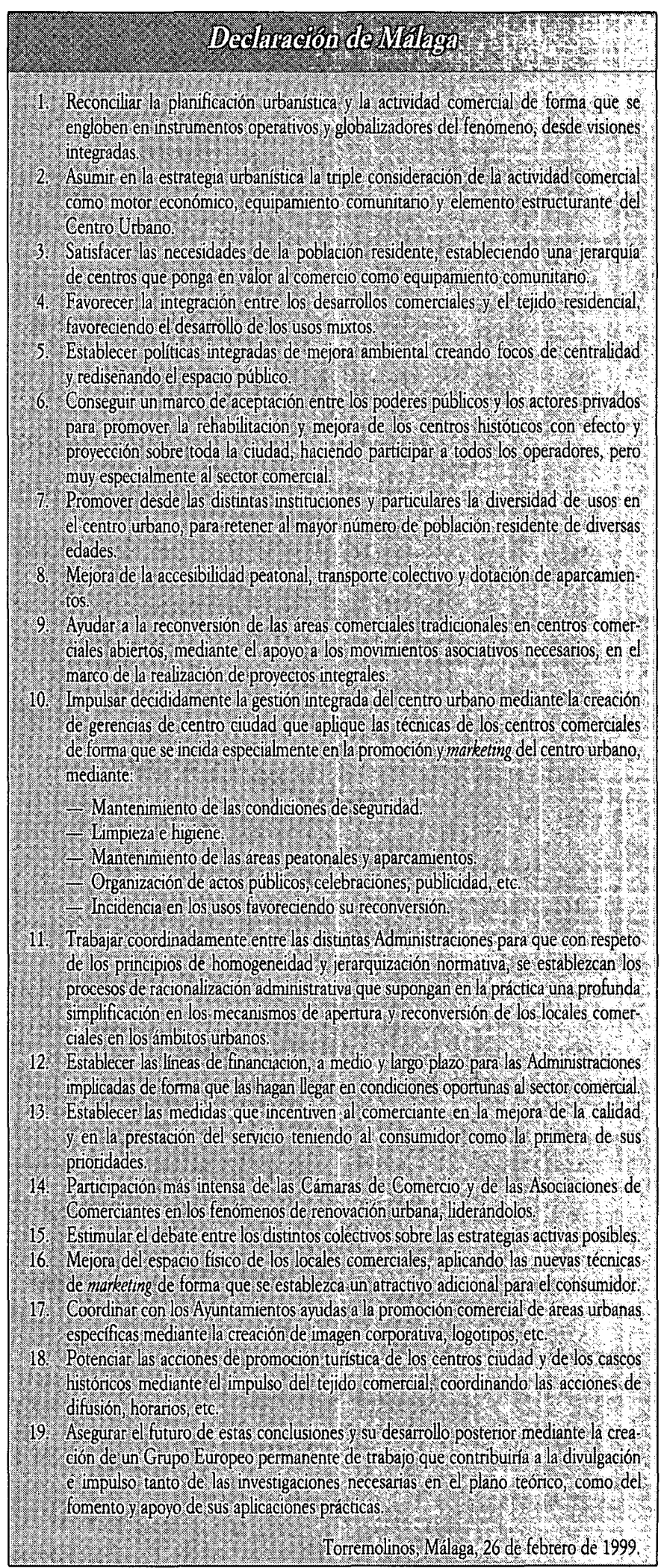

Para poder presentar «buenas prácticas» en el transcurso del Congreso, Francia realizó dos estudios sobre políticas comerciales y desarrollo urbano, uno referido a diez ciudades 
francesas y el otro a trece ciudades de otros países europeos. Además, se planteó la inclusión de cuatro «talleres» para la presentación de algunas de estas «mejores prácticas». Para seleccionar los casos a exponer en los talleres se abrió un periodo de presentación de actuaciones por parte de las Administraciones Públicas, empresas públicas, asociaciones o empresas del comercio o de urbanismo, etc., dirigida a todos los Estados europeos, no sólo a los miembros de la UE. Estos cuatro talleres se refieren a:

Taller A: «La estrategia de las ciudades», orientado hacia el análisis de las estrategias de renovación urbana desarrolladas por los ayuntamientos y que se basan en el papel del comercio para alcanzar los objetivos previstos. Como factores a analizar dentro de las buenas prácticas se han considerado la definición de una política global en la que el comercio sea un factor destacado, la importancia que se le concede en las distintas fases del proceso de renovación urbana, la consideración de los tipos de comercio más eficaces para lograr los objetivos que se persiguen y la localización según tipos y los medios que desarrollan las administraciones municipales para hacer más atractivas las áreas comerciales que han sido objeto de la renovación.

Taller B: «Las estrategias de las empresas». Se trata de definir cuáles son las mejores formas de actuación de los distintos tipos de empresas comerciales para obtener el mayor beneficio de las operaciones de renovación urbana. Además, se estudiarán las condiciones de competencia entre las empresas comerciales, según su localización y según su dimensión, analizándose las condiciones de equilibrio entre los objetivos del planeamiento urbano y el desarrollo de un comercio competitivo y que cubra las necesidades de la población.

Taller C: «Proyectos y financiación: cooperación entre los sectores público y privado»: Se trata de analizar las formas más eficaces de colaboración entre los operadores públicos y privados para la financiación de acciones de renovación urbana. Se intenta, a la vista de las buenas prácticas expuestas, dar respuesta a preguntas tales como cuáles son los distintos operadores que deben participar en la cofinanciación, las condiciones que deben darse para que la colaboración sea eficaz y los montajes jurídicos necesarios para conseguirlo.

Taller D: «Los instrumentos de gestión urbana, palancas de las operaciones de renovación»: Se trata de identificar los instrumentos más útiles de que disponen los ayuntamientos para las operaciones de renovación urbana en las que el comercio tenga un papel relevante, instrumentos tales como adecuación de usos, facilitar el acceso a los no residentes, mejorar las condiciones de seguridad y limpieza, hacer más atractivas las áreas seleccionadas mediante el establecimiento de otro tipo de actividades, especialmente culturales o de ocio, etc.

Y como primer resultado, después de estos estudios y de la selección de actuaciones, se llegó a la conclusión de que las ciudades europeas son muy distintas unas de otras, y que no toda actuación que se ha mostrado eficaz en una puede ser exportada a las demás. Merecen también destacarse dos consideraciones que con carácter general han sido puestas de relieve a lo largo de todo el Congreso: no cabe ya referirse a un centro urbano único, sino que debe hablarse de varios centros o barrios comerciales en nuestras ciudades de un cierto tamaño, y no se puede ya seguir hablando de un enfrentamiento entre pequeño comercio y gran superficie, sino que ambos deben unirse para aumentar y potenciar el atractivo del comercio urbano.

En el análisis que se ha realizado en los debates del Congreso sobre las estrategias de las ciudades, ha quedado de manifiesto la necesidad de una visión unitaria de lo que se pretende y compartida por todos los actores que intervienen en el proceso. Las administraciones nacionales o regionales deben actuar unidas a la local, quien a su vez debe animar a los actores privados, el comercio minorista, a «apropiarse de la idea». Y de esta forma, establecer la cooperación, el «partenariado», entre los sectores público y privado, de una manera formal o informal, pero con una clara constancia de que la acción a realizar va a suponer un beneficio colectivo y va a implicar unos riesgos, aceptando que beneficios y riesgos deben ser compartidos por todos.

En ciertos tipos de estrategias urbanas debe superarse la confrontación tradicional entre centro y periferia, para reforzar el concepto de atracción global de la aglomeración; los distintos polos comerciales de las grandes ciudades deben interconectarse entre sí para potenciar un atractivo global, y no limitado a áreas reducidas de la ciudad.

Sin embargo, se ha destacado el especial significado del centro tradicional de la ciudad. El reforzar la atracción de este centro debe ser el principal objetivo, considerando que el proyecto urbano y el proyecto de equipamiento comercial son claramente interdependientes, por lo que debe buscarse una articulación estrecha entre ambos. Es de sobra sabido y repetido que los buenos emplazamientos hacen buenos comercios. No puede decirse lo mismo a la inversa. Por eso es necesario el estudio previo de la zona, y hacerla atractiva, accesible y segura para aumentar el flujo de visitantes y potenciales clientes. Una vez dado este primer paso por las administraciones locales, el buen comercio debe potenciar ese atractivo de la zona, colaborando a que su entorno sea un «buen emplazamiento». Para asegurar el éxito, dos condiciones son necesarias: alentar la modernización de la actividad comercial y también potenciar la diversificación de la oferta.

La modernización del comercio no es más que una demanda del consumidor; sus gustos evolucionan cada vez más deprisa, pidiendo unos productos distintos y unos servicios cada vez mejores. El mantenimiento de una oferta estática y de unos servicios anticuados es incompatible con el fortalecimiento de 
la actividad comercial. Esta modernización exige inversiones para mejorar la calidad y variedad de la oferta, de manera que los consumidores tengan más donde elegir, tengan mejores servicios y una mayor comodidad en sus compras.

El buen emplazamiento debe contar además con el complemento de otras actividades. Se ha considerado, con carácter general en todos los casos presentados, la importancia de instalar en los centros urbanos a potenciar toda una serie de servicios que atraigan potenciales compradores a la zona, instalando servicios públicos y equipamientos culturales o de ocio, rehabilitando edificios históricos o con cierto atractivo cultural e impulsando actos lúdicos, al tiempo de dinamizar la interrelación entre el comercio y el hábitat, recalificando y diversificando la oferta de locales. Y, cómo no, garantizar la seguridad y la limpieza del área.

La creación de islas peatonales en los centros de ciudad puede ser también una herramienta eficaz para su dinamización comercial. Pero puede resultar contraproducente si la peatonalización no va unida a la facilidad de acceso para los residentes en otras zonas. La eficacia de una isla peatonal viene condicionada por la existencia de áreas de aparcamiento en sus inmediaciones, a las que se pueda llegar con facilidad, y la existencia de un transporte colectivo eficaz, cómodo y seguro, ya que, de otra forma, la isla peatonal se convertiría en una forma de dificultar la entrada de compradores no residentes. Pero también en esa isla peatonal hay que garantizar que las operaciones de carga y descarga no se van a ver dificultadas.

Se ha considerado también la necesidad y eficacia de la gerencia única, los «town centre management», para efectuar la gestión de lo cotidiano en la que están implicados todos los afectados al centro ciudad, realizando una gestión que pudiera equipararse a la que se realiza en los centros comerciales que proliferan en la periferia de las ciudades europeas, para dar una respuesta eficiente a lo que espera encontrar el visitante y promover acciones de marketing y promoción unitaria del centro urbano. Para facilitar el éxito de la gerencia unitaria es importante potenciar las asociaciones de comerciantes e integrar en la asociación espacial tanto al pequeño comerciante aislado como al comercio organizado, las franquicias y a las grandes superficies existentes en el área.

Se ha puesto de manifiesto a lo largo del Congreso la importancia del comercio como rehabilitador de barrios degradados. No se trata ya sólo de potenciar el centro histórico de la ciudad, haciendo aumentar su oferta comercial, sino también, en la misma línea, el potenciar otros «centros» de las grandes ciudades, defendiendo el comercio de barrio y el atractivo integral de éstos. Pero, además, en los barrios degradados el comercio puede jugar un papel decisivo en su reestructuración. La Comisión Europea apuesta decididamente en esta línea, con el apoyo de fondos estructurales del Programa Urban. La remodelación de un barrio degradado realizada por las administraciones públicas, que puede llegar incluso a la demolición parcial del mismo, encuentra en la potenciación de la actividad comercial una importante ayuda para reacreditar de nuevo el barrio, unida al desarrollo de otras acciones de atracción para generar un importante flujo de no residentes. El alcanzar una masa crítica de comercios en el barrio rehabilitado, evitando la permanencia de locales vacíos, es necesario para evitar su nueva degradación.

$\mathrm{Al}$ analizar la estrategia de las empresas, se ha puesto de relieve la importancia de las locomotoras para la revitalización de un área comercial. Las grandes enseñas ejercen un importante papel de atracción de compradores, mientras que el comercio independiente debe especializarse y encontrar su adecuado nicho de mercado para beneficiarse del flujo de visitantes del barrio atraídos por la gran superficie. Si en algunos casos específicos este papel de locomotora o reclamo de visitantes puede ser ejercido por algún tipo de actividad ajena al sector comercial, como puede ser algún museo o algún centro cultural o de ocio, con carácter general, las grandes superficies o los centros comerciales cerrados deben realizar esta función de locomotoras para el comercio urbano.

Se viene apreciando desde mediados de los años noventa un retorno del interés de las grandes empresas de distribución por los centros urbanos, tal vez con formatos más pequeños. La estrategia de revitalización de los centros urbanos en declive debe aprovechar este interés para facilitar la introducción de estas nuevas locomotoras y de enseñas comerciales con fuerte notoriedad, especialmente en materias de equipamiento personal, cultural o de ocio. Se ha puesto de relieve en los casos estudiados que son las colectividades locales, las administraciones municipales, las que disponen de herramientas para hacer viable la llegada de estos nuevos operadores a los centros en declive, facilitando la realización de las intervenciones inmobiliarias y de acondicionamiento de las zonas elegidas para facilitar el acceso de estas nuevas locomotoras.

En los debates del Congreso ha quedado patente que las asociaciones de comerciantes no han sido los motores de los proyectos de introducción de «locomotoras», bajo la forma de centro comercial o de grandes o medianas superficies explotadas conjuntamente, pero sí que se han producido unas importantes sinergias entre los centros comerciales/grandes superficies y el comercio independiente o en cadenas cuando éstos han sabido adaptarse y ofrecer una especialización y servicio de calidad a los clientes, ofreciendo una imagen conjunta del centro de ciudad como zona de atractiva habitabilidad y destacando como lugar de compras placenteras, en oposición a las «compras por necesidad» que se realizan en los equipamientos comerciales de la periferia.

Se ha destacado el tipo de establecimiento que debe servir de locomotora según el lugar donde haya de instalarse. Para 
los centros históricos de las ciudades son las enseñas acreditadas de equipamiento personal las que deben actuar como atracción de clientes, así como también las de productos culturales y de ocio, como libros o discos. Por el contrario, para los barrios comerciales distintos del centro histórico y especialmente para la rehabilitación de barrios degradados, las grandes y medianas superficies de alimentación están llamadas a ejercer esa acción motriz del comercio tradicional que debe implantarse a su alrededor.

Una vez más, y en el taller específico al respecto, se puso de relieve la necesidad de la cooperación entre el sector público y el sector privado. El desarrollo urbano debe promoverse por la administración local, iniciándolo con un análisis global de la situación y necesidades de la ciudad. Pero, una vez definida la situación y objetivos a alcanzar, debe implicarse a todos las sectores y agentes económicos y sociales de la ciudad. La inversión pública es necesaria, y ésta debe servir como palanca para activar y fomentar la inversión privada. El impulso de las asociaciones zonales, la concentración o provisión de espacio para la instalación de las locomotoras, la definición y ordenación de las zonas peatonales, la creación del gerente único, deben ser fomentados por la administración local, dialogando y contando con la participación y apoyo del sector comercial.

$\mathrm{Ha}$ quedado de relieve que uno de los principales problemas con que se encuentran los centros tradicionales o históricos de las ciudades es el desplazamiento de la población desde ese centro hacia la periferia, donde han aparecido nuevas formas comerciales que han alejado a los consumidores de los centros urbanos. Ante esto, y como conclusión de las «buenas prácticas» que han sido presentadas, se llega a la necesidad de aplicar la regla de las 4 A: Animación, Accesibilidad, Atractivo y Acción. La declaración de Lille se concretó en los tres artículos que se recogen a continuación.

\section{Drecharacion de LATle}

La gran diversidad de nuestras ciudades nos impide dar un modelo europeo único de desarrollo urbano. Por ello, el Congreso de Lille se ha fijado como objetivo identificar los mejores métodos, las «buenas prácticas» para el comercio en la renovación urbana.

Tres criterios para el éxito han sido destacados:

1. Asociar, desde el comienzo de la operación, a los comerciantes de los centros de las ciudades y de los barrios, con el fin de obtener su adhesión al proyecto.

2. Tener en cuenta las necesidades reales de los actores del comercio urbano y de los consumidores, descartando la importación artificial de modelos de desarrollo urbano que hayan podido tener éxito en otros lugares:

3. Tanto en la reflexión como en la acción, ir más allá de las cuestiones comerciales y urbanisticas para desarrollar un enfoque global tanto en el plano territorial como en el económico.

Lille, 17 de noviembre de 2000
Como corolario de estos tres artículos, «se formulan las siguientes recomendaciones para los actores de la renovación urbana, que son las empresas de comercio y las autoridades locales:

Para las empresas del comercio:

- Proponer soluciones creativas e innovadoras.

- Desarrollar conceptos innovadores de almacén en centro ciudad y también en los barrios y en la periferia.

- Participar en la reconversión y en la renovación de los centros comerciales $y$ de las grandes superficies de alimentación en los barrios en dificultad.

- Desarrollar una presencia activa en las instancias de concertación.

- Relacionar las estrategias de marketing con los proyectos urbanos.

\section{Para las autoridades locales:}

- Realizar, previamente a las actuaciones, los estudios necesarios para tomar una decisión.

- Velar por una articulación estrecha entre el proyecto urbano y el proyecto comercial.

- Mantener un diálogo continuo desde el principio de los estudios hasta la finalización de las actuaciones, con los colaboradores económicos.

- Asociar los actores económicos a los trabajos de los órganos de cooperación.

- Incluir a los operadores económicos en la gestión comercial y urbana del centro de ciudad.

Para alcanzar el éxito en nuestras ciudades, existen numerosas berramientas en común:

- El control de las decisiones administrativas que permitan rentabilizar una oferta territorial adaptada a las necesidades locales.

- El control de los planes de transporte y aparcamiento.

- La facultad de reformar los espacios públicos, proteger la arquitectura local e intervenir sobre el bábitat.

- La responsabilidad del desarrollo de servicios públicos locales y de equipamientos educativos, culturales y sociales.

- La promoción de las ventajas de la ciudad.»

Volviendo a las conclusiones que se englobaron en la declaración de Málaga, nos encontramos con que muchas de ellas aparecen de manera recurrente a lo largo de las sesiones del Congreso de Lille como buenas prácticas presentadas en los distintos talleres o recogidas de los estudios realizados sobre las ciudades. En concreto, se vuelve a incidir en aspectos tales como la mejora de la accesibilidad global a los centros urbanos, la promoción de los diversos usos de los centros ciudad, la necesidad de una gestión integrada, de coordinación en la toma de decisiones y planificación, de la mejora de la calidad y de los servicios del comercio y en la mejora de la finan- 
ciación, buscando el partenariado entre los sectores públicos y privados.

Otros, como la necesidad de las locomotoras y el potenciar no sólo el centro histórico, sino los distintos barrios, potenciando el atractivo global y la intercomunicación entre los distintos polos comerciales de la aglomeración, aparecen por primera vez a lo largo de este Congreso.
Entre el 4 y 6 de septiembre del año 2002, en la ciudad alemana de Leipzig, el Tercer Congreso Europeo de Comercio y Ciudad nos ofrecerá la posibilidad de comprobar la vigencia de estos principios y de conocer qué otras acciones son aconsejadas para la revitalización del centro ciudad, y del comercio de centro ciudad, en las ciudades del continente más urbanizado del mundo.

* Subdirector General de Estudios y Modernización del Comercio Interior. Dirección General de Política Comercial. Ministerio de Economía.

\section{Bibliografía}

Libro Verde del Comercio, Comisión Europea.

Libro Blanco del Comercio, Comisión Europea.

Declaración de Málaga, Dirección General de Comercio Interior, Ministerio de Economía y Hacienda.

Le Commerce et la Ville. Politiques commerciales et développement urbain dans les pays de l'Union européenne, Ministère de l'Economie, des Finances et de l'Industrie, Direction des Entreprises Commerciales, Artisanales et de Services.
Le Commerce et la Ville. Politiques commerciales et développement urbain dans les villes françaises, Ministère de l'Economie, des Finances et de l'Industrie, Direction des Entreprises Commerciales, Artisanales et de Services.

Discours de clôture et Declaration de Lille, Secrétaire d'État aux PME, au commerce, à l'artisanat et à la consommation. 